\title{
Extended lymphadenectomy benefits patients with borderline resectable pancreatic head cancer-a single-center retrospective study
}

\author{
Jing Wang", Shao-Cheng Lyu", Ji-Qiao Zhu", Xian-Liang Li, Ren Lang, Qiang He \\ Department of Hepatobiliary and Pancreaticosplenic Surgery, Beijing Organ Transplant Center, Beijing Chaoyang Hospital, Capital Medical \\ University, Beijing, China \\ Contributions: (I) Conception and design: JQ Zhu, SC Lyu, J Wang; (II) Administrative support: R Lang, Q He; (III) Provision of study materials \\ or patients: J Wang, R Lang; (IV) Collection and assembly of data: J Wang, SC Lyu; (V) Data analysis and interpretation: SC Lyu, XL Li; (VI) \\ Manuscript writing: All authors; (VII) Final approval of manuscript: All authors. \\ \#These authors contributed equally to this work. \\ Correspondence to: Ren Lang, MD; Qiang He, MD. No. 8 Gongtinan Road, Chaoyang District, Beijing 100020, China. \\ Email: dr_langren@126.com; heqiang349@sina.com.
}

Background: Whether standard lymphadenectomy or extended lymphadenectomy should be performed is still under debate during pancreaticoduodenectomy (PD). We aimed to compare their morbidity and mortality rates among patients with pancreatic head cancer (PHC).

Methods: In this retrospective study, a total of 322 patients were enrolled. According to the scope of intraoperative lymph node dissection, patients were divided into extended lymphadenectomy group $(n=120)$ and standard lymphadenectomy group $(n=202)$. Based on the resectability of the tumor, there were 198 cases of resectable PHC and 124 cases of borderline resectable PHC, respectively, in which further stratified analysis was carried out according to the extent of lymph node dissection.

Results: All patients completed the operation successfully, with a perioperative morbidity rate of $27.9 \%$ and mortality rate of $0.9 \%$. As for the overall patients, patients in the extended lymphadenectomy group had higher neutrophil-to-lymphocyte ratio (NLR), longer operation time, more intraoperative blood loss, lymph node dissection and patients with borderline resectable pancreatic head cancer (BRPHC) $(\mathrm{P}<0.05)$. The 1-, 2and 3-year overall survival rates of patients with extended lymphadenectomy and standard lymphadenectomy were $71.9 \%, 50.6 \%, 30.0 \%$ and $70.0 \%, 32.9 \%, 21.5 \%$, respectively $(\mathrm{P}=0.068)$. With regards to patients with BRPHC, the number of lymph node dissection in the extended lymphadenectomy group was more $(\mathrm{P}<0.05)$, and the 1-, 2- and 3-year overall survival rates of patients with extended lymphadenectomy and standard lymphadenectomy were $60.7 \%, 43.3 \%, 27.4 \%$ and $43.2 \%, 17.7 \%, 17.7 \%$, respectively (P=0.007).

Conclusions: Patients with BRPHC tended to have vast lymph node metastasis. Extended lymphadenectomy can improve their long-term survival.

Keywords: Pancreaticoduodenectomy (PD); pancreatic head cancer (PHC); standard lymphadenectomy; extended lymphadenectomy; prognosis

Submitted Mar 30, 2021. Accepted for publication Sep 09, 2021.

doi: 10.21037/gs-21-201

View this article at: https://dx.doi.org/10.21037/gs-21-201

(c) Gland Surgery. All rights reserved. 


\section{Introduction}

Pancreatic cancer is becoming one of the most common cause of cancer mortality, whose incidence has increased in the past decade despite a decrease in incidence rates for other cancers (1). Although considerable progress has been made to improve the survival rate surgical resection remains the only potential curative method for patients with pancreatic cancer (2-4). Notably, lymphadenectomy, which is a powerful prognostic factor after pancreaticoduodenectomy (PD) that is independent of the histology, has been strongly suggested to achieve the potential cure when pancreatic cancer is suspected $(5,6)$. At present, whether standard lymphadenectomy or extended lymphadenectomy should be performed is still under debate. Ishikawa et al. reported extended clearance of regional lymph nodes and soft tissue could significantly prolong the survival without increasing the morbidity rate (7). In contrast, Jang et al. found extended resection failed to improve oncologic efficacy or long-term survival compared with standard resection (8). Whereas, Pedrazzoli et al. did notice a trend toward longer survival after extended lymphadenectomy instead of standard lymphadenectomy in spite of similar overall survival rates between the two groups (9). Therefore, it is the surgeons' major concern to determine their efficacy among these patients.

Moreover, previous studies focused on all patients with pancreatic head cancer without distinguishing their stages, who actually had different tumor biology and lymph node status $(10,11)$. Since it is really difficult to thoroughly assess the extent of positive lymph nodes in the preoperative and intraoperative periods due to currently limited clinical technology, we conducted a retrospective analysis in terms of resectability including resectable and borderline resectable pancreatic head cancer (BRPHC) recommended by the National Comprehensive Cancer Network (NCCN) guidelines (12). We aimed to compare the morbidity and mortality rates between extended lymphadenectomy and standard lymphadenectomy.

We present the following article in accordance with the STROBE reporting checklist (available at https://dx.doi. org/10.21037/gs-21-201).

\section{Methods}

\section{Ethics approval and consent to participate}

The study was conducted in accordance with the Declaration of Helsinki (as revised in 2013). The study was approved by the Ethics Committee of Beijing Chaoyang Hospital (No. 2020-D.-309-2) and individual consent for this retrospective analysis was waived.

\section{Inclusion and exclusion criteria}

The data of patients with pancreatic head carcinoma (PHC) who underwent PD in the Department of Hepatobiliary surgery of Beijing Chaoyang Hospital from January 2010 to December 2019 were analyzed retrospectively. According to the relevant inclusion/exclusion criteria and NCCN guidelines, 322 patients with PHC were enrolled [including 198 patients with resectable pancreatic head cancer (RPHC) and 124 patients with BRPHC].

Inclusion criteria: (I) patients with $\mathrm{PHC}$ who underwent PD from January 2010 to December 2019; (II) aged 20 to 85 years old; (III) assessed as RPHC or BRPHC according to the NCCN guidelines; (IV) en bloc resection of tumor during operation; (V) postoperative pathology confirmed pancreatic ductal adenocarcinoma; (VI) the mode of operation and treatment strategy obtained the informed consent of patients and their families.

Exclusion criteria: (I) unresectable condition or metastasis found during surgery; (II) surgical rule violation; (III) pathologic diagnosis other than conventional ductal adenocarcinoma; (IV) postoperative follow-up data were incomplete or lost to follow-up (Figure 1).

\section{Patients grouping and definition}

According to the NCCN guidelines, the patients were divided into 198 cases of RPHC and 124 cases of BRPHC. RPHC was defined as the tumor did not invade the celiac artery or vein and had no distant metastasis and BRPHC included 45 cases of tumor invading the confluence of portal vein ( 2 cases contacting celiac trunk but invading $<180$ degrees), 42 cases of tumor invading superior mesenteric vein ( 3 cases contacting superior mesenteric artery but invading $<180$ degrees), 37 cases invading portal vein ( 2 cases contacting common hepatic artery but invading $<180$ degrees) (Figure 2).

The lymph node grouping is named according to the Japanese Society of Hepato-Biliary-Pancreatic Surgery (13). According to the range of intraoperative lymph node dissection, the patients were divided into extended lymph node dissection group and standard lymph node dissection group, and differences in the extent of resection are summarized in Table 1. Surgical outcomes such as 


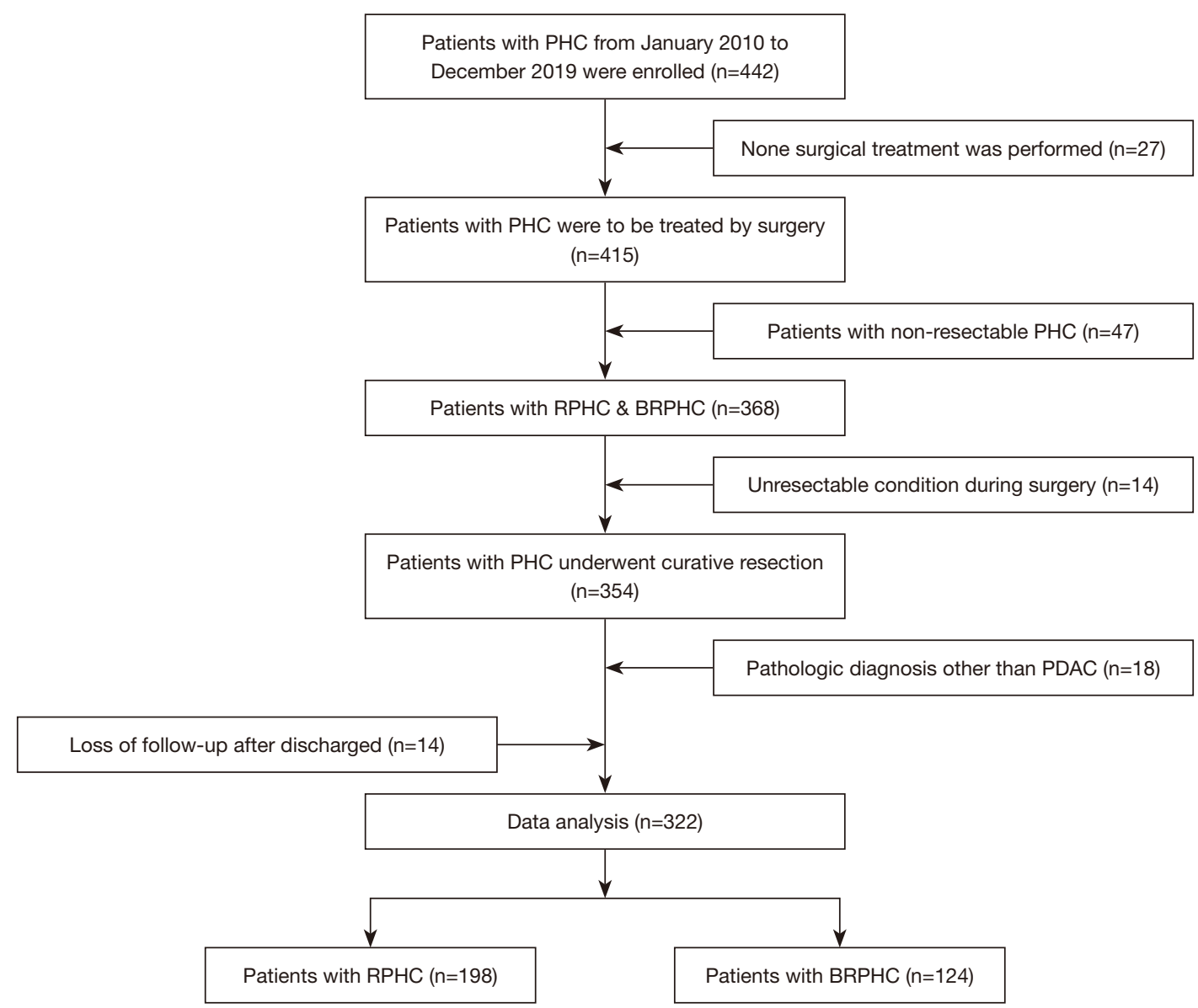

Figure 1 Screening flow chart. BRPHC, borderline resectable pancreatic head cancer; PDAC, pancreatic ductal adenocarcinoma; PHC, pancreatic head cancer; RPHC, resectable pancreatic head cancer.

intraoperative blood loss, operation time, curative resection (R0), postoperative complication and so forth were collected. R0 was defined as a specimen with clear resection margins, tumor cell was not found within $1 \mathrm{~mm}$ distance from margin. Postoperative complications were graded according to the Clavien-Dindo classification (14). Drain amylase of $>3$ times serum amylase after the third postoperative day, as defined by International Study Group of Pancreatic Surgery (ISGPS), was defined as pancreatic fistula (15).

\section{Surgical techniques}

Some studies have shown that enlarged dissection of retroperitoneal lymphoid tissue and nerve plexus was performed on the basis of radical PD for pancreatic head cancer. The postoperative histopathology showed frequent lymph node metastasis from celiac trunk to superior mesenteric artery. It is closely related to the prognosis of pancreatic cancer, suggesting that extended lymph node dissection can benefit the survival of patients. Therefore, on the basis of standard dissection range, we dissected the lymph nodes behind the hepatic artery [8p], around the celiac artery [9], around the proper hepatic artery [12a], behind the portal vein [12p], around the superior mesenteric artery [14a-d], and around the abdominal aorta [16a2c16b1]. The scope of dissection was up to the hepatic hilum, down to the beginning of the inferior mesenteric artery, left to the left edge of the abdominal aorta, and right to the right renal hilum to complete the dissection of lymph, nerve and connective tissue in this area.

\section{Index analysis and follow-up strategy}

The clinical and pathological data were extracted from the 

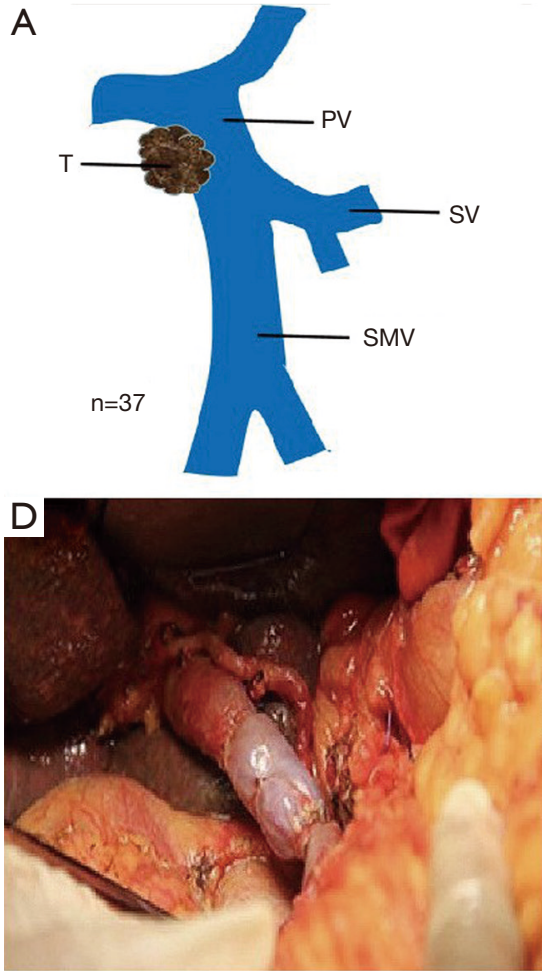

B
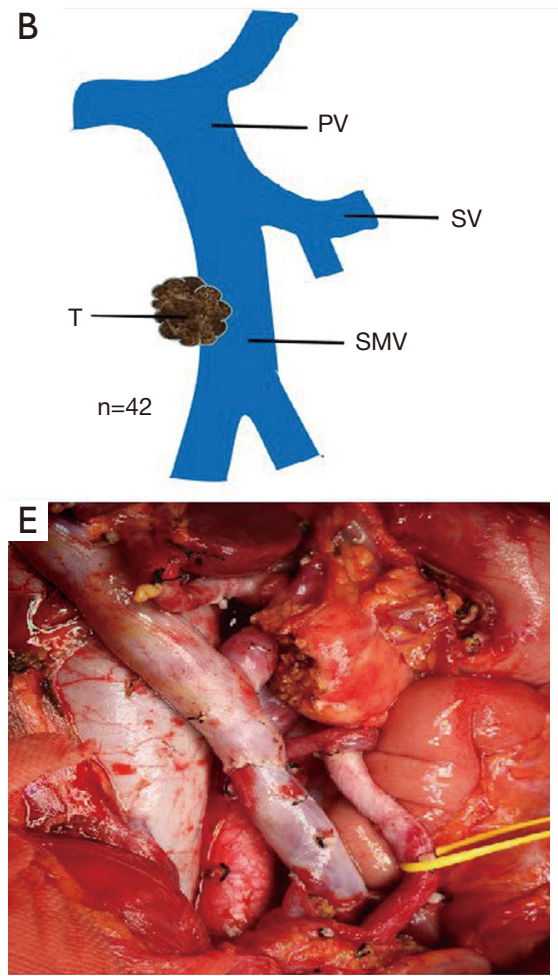

C

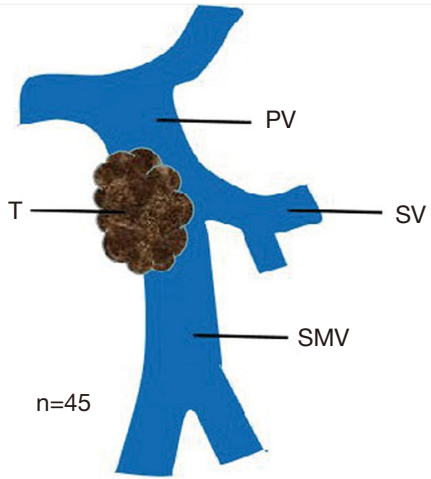

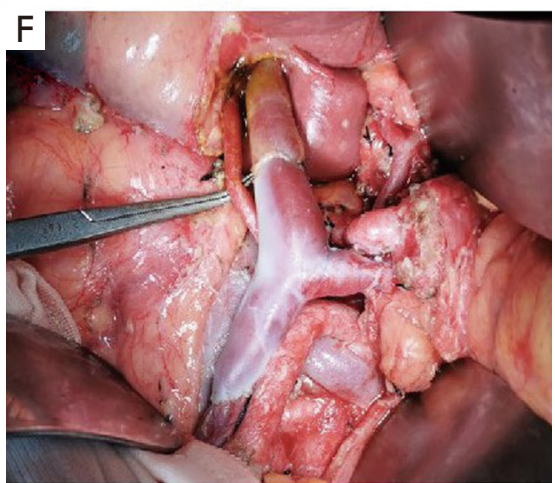

Figure 2 Venous invasion of borderline resectable pancreatic head cancer. (A) Tumor invades portal vein ( $\mathrm{n}=37$ ); (B) tumor invades superior mesenteric vein $(n=42)$; (C) tumor invades the confluence of portal vein $(n=45)$; (D) postoperative effect of tumor invasion on portal vein; (E) postoperative effect of tumor invasion on superior mesenteric vein; (F) postoperative effect of tumor invasion on the confluence of portal vein. PV, portal vein; SV, spleen vein; SMV, superior mesenteric vein.

medical records, and the perioperative data of different groups were compared. The frequency of postoperative follow-up: 1 and 3 months after operation, once every 3 months within 2 years, and once every half a year for more than 2 years. The end points of follow-up were tumor recurrence and death. The contents of follow-up mainly included blood examination (blood routine, blood biochemistry, tumor markers, etc.) and imaging examination [abdominal enhanced computed tomography (CT), pulmonary CT, enhanced CT, etc.], follow-up treatment, tumor recurrence and survival, and compared the long-term prognosis of patients in different groups.

\section{Statistical analysis}

Results are presented by mean \pm standard deviation in accordance with normal distribution and by median [interquartile range (IQR)] in non-normal distribution. Nominal data were compared using $\chi^{2}$ tests and continuous variables using Student's $t$-tests. Survival outcomes were calculated using the Kaplan-Meier method and compared using the log-rank test. Only variables statistically significant by univariate analysis were included in the multivariate analysis, which was performed using a Cox proportional hazards regression model. All statistical analyses were performed using SPSS, version 22.0 (IBM), with two-sided $\mathrm{P}$ values less than 0.05 considered statistically significant.

\section{Results}

\section{Clinical characteristics and outcomes}

A total of 322 patients with pancreatic head cancer were identified. Three patients died in perioperative period, with a perioperative mortality rate of $0.9 \%$, and the reason for the death was grade $\mathrm{C}$ pancreatic fistula complicated with abdominal hemorrhage. One-hundred and twenty patients with extended dissection and 202 patients with standard dissection were performed according to the extent of lymph node dissection. According to the location of the 
Table 1 Summary of difference of the extent of surgical dissection according to the study group

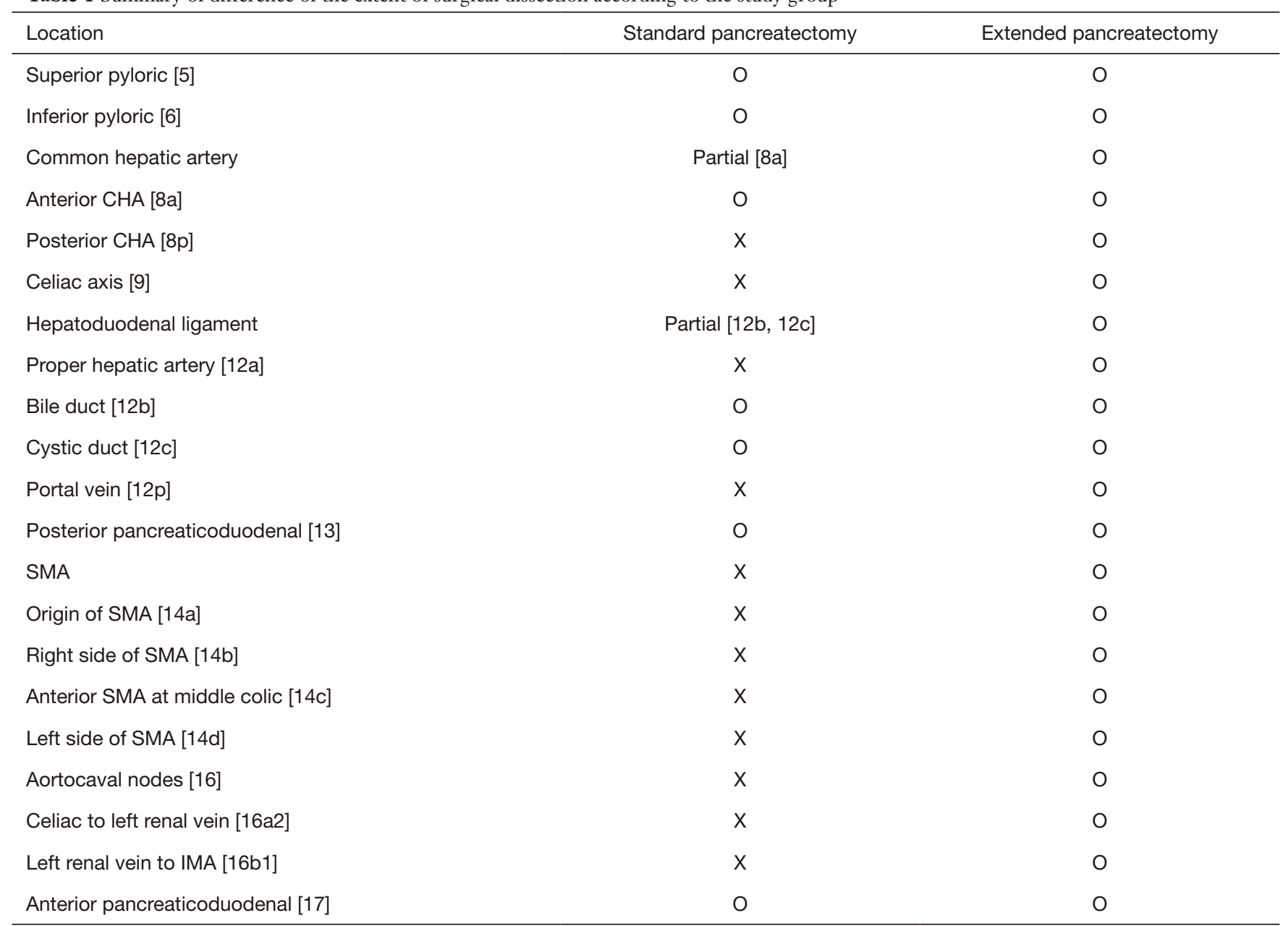

$\mathrm{O}$ indicates dissected; X, not dissected. CHA, common hepatic artery; IMA, inferior mesenteric artery; SMA, superior mesenteric artery.

vein invaded by the tumor, 124 patients with borderline resectability underwent allogeneic vascular replacement after invasive vascular resection in 87 cases, end-to-end anastomosis in 25 cases, and direct suture after wedge resection in 12 cases. The intraoperative blood loss was 500 (IQR, 400, 1,000) $\mathrm{mL}$, blood transfusion was performed in 108 patients $(33.5 \%)$, and the operation time was $9.8 \pm 2.9 \mathrm{~h}$.

\section{Perioperative condition}

Postoperative complications occurred in 90 cases, with a complication rate of $27.9 \%$. Biochemical fistula occurred in 29 cases $(9.0 \%), \mathrm{B}$ pancreatic fistula in 8 cases (2.5\%), C pancreatic fistula in 5 cases $(1.6 \%)$, diarrhea in 32 cases $(9.9 \%)$, DGE (disturbance of gastric emptying) in
30 cases $(9.3 \%)$, abdominal infection in 17 cases (5.3\%), abdominal hemorrhage in 13 cases $(4.0 \%)$, biliary fistula in 6 cases $(1.9 \%)$, pulmonary infection in 5 cases $(1.6 \%)$, gastrointestinal bleeding in 3 cases $(0.9 \%)$. There were 2 cases of intestinal fistula $(0.6 \%), 2$ cases of portal vein thrombosis $(0.6 \%)$ and 1 case of pulmonary embolism $(0.3 \%)$. The median postoperative hospital stay was 17 (IQR, 14, 23) days (range, 7-106 days).

All patients were confirmed as pancreatic ductal adenocarcinoma by pathological examination. Among them, the degree of tumor differentiation: moderately differentiated in 209 cases $(64.9 \%)$, poorly differentiated in 93 cases $(28.9 \%)$, and highly differentiated in 20 cases (6.2\%); tumor size: $3.8 \pm 1.6 \mathrm{~cm}$ (range, $1.0-10.0 \mathrm{~cm}$ ). The average number of lymph nodes detected was $21.2 \pm 12.1$ (range, 5-86), positive lymph nodes in 208 cases (64.6\%), 

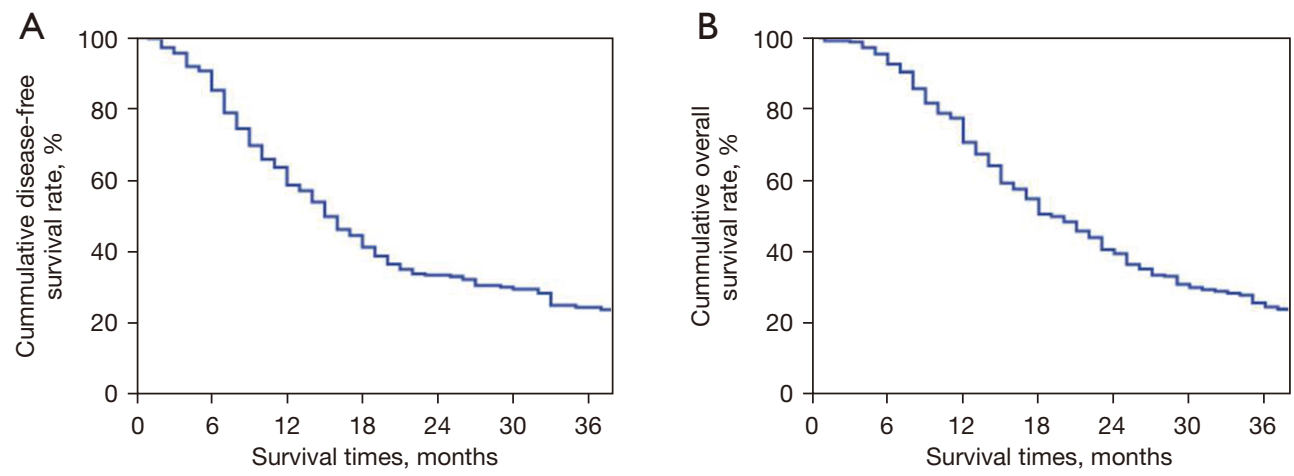

\begin{tabular}{|c|c|c|c|c|c|c|c|}
\hline \multicolumn{4}{|c|}{ DFS } & \multicolumn{4}{c|}{ OS } \\
\hline Year & 1 & 2 & 3 & Year & 1 & 2 & 3 \\
\hline Rate & $58.7 \%$ & $33.6 \%$ & $24.5 \%$ & Rate & $70.7 \%$ & $39.5 \%$ & $24.6 \%$ \\
\hline
\end{tabular}

Figure 3 Overall long-term prognosis of patients. (A) Disease-free survival (DFS) curve of patients with pancreatic head cancer; (B) overall survival (OS) curve of patients with pancreatic head cancer.

positive number of lymph nodes in 2 [IQR, 0, 4] (range, $0-22$ ), and MLNR (metastatic lymph node ratio) was 0.09 [IQR, 0, 0.24] (range, 0-0.76). Radical resection (R0) was achieved in 301 cases $(93.5 \%)$, pancreatic cutting edge was positive in 8 cases $(2.5 \%)$, pancreatic circumferential margin was positive in 7 cases $(2.2 \%)$, pancreatic uncinate process margin was positive in 4 cases $(1.2 \%)$, and portal sulcus margin was positive in 2 cases $(0.6 \%)$.

\section{Survival data and recurrence}

The follow-up period was up to June 2020, with a median follow-up period of 51 months. During this period, 107 patients (33.2\%) received adjuvant chemotherapy for 1 to 8 cycles. The overall median disease-free survival time of the patients was 15 months (Figure $3 A$ ) and the overall median survival time of the patients was 19 months (Figure 3B).

\section{Comparison of patients with different lymph node dissection groups}

The comparison of perioperative general data between the extended dissection group and the standard dissection group was shown in Table 2. It can be seen that the patients in the extended dissection group had higher neutrophilto-lymphocyte ratio (NLR), longer operation time, more intraoperative blood loss, more lymph node dissection and more patients with borderline resectability $(\mathrm{P}<0.05)$. The comparison of postoperative morbidity between two groups was shown in Table 3. It can be seen that the incidence of diarrhea in the extended dissection group was higher than that in the standard dissection group $(\mathrm{P}<0.05)$ and there were no statistically significant differences in other perioperative complication rates and mortality rate $(\mathrm{P}>0.05)$.

The median disease-free survival time of patients with extended lymph node dissection and standard lymph node dissection was 16 and 15 months, respectively. The 1-, 2and 3 -year disease-free survival rates were $61.4 \%, 40.7 \%$, $29.0 \%$ and $57.2 \%, 29.3 \%$ and $20.8 \%$, respectively $(\mathrm{P}=0.125$, Figure $4 A$ ). And the overall median survival time of patients with extended lymph node dissection and standard lymph node dissection was 25 and 18 months, respectively. The 1 -, 2- and 3-year overall survival rates were $71.9 \%, 50.6 \%$, $30.0 \%$ and $70.0 \%, 32.9 \%, 21.5 \%$, respectively $(\mathrm{P}=0.068$, Figure $4 B)$.

\section{Comparison of patients with RPHC by different lymph node dissection groups}

The comparison of perioperative general data between the extended dissection group and the standard dissection group was shown in Table 4. It can be seen that the NLR value of the extended dissection group was higher, the operation time was longer, and the number of lymph node dissection was more than that of the standard dissection group $(\mathrm{P}<0.05)$. The comparison of postoperative complications between two groups was shown in Table 5. It can be seen that the incidence of diarrhea in the extended lymph node 
Table 2 Demographic and pathologic findings between two groups

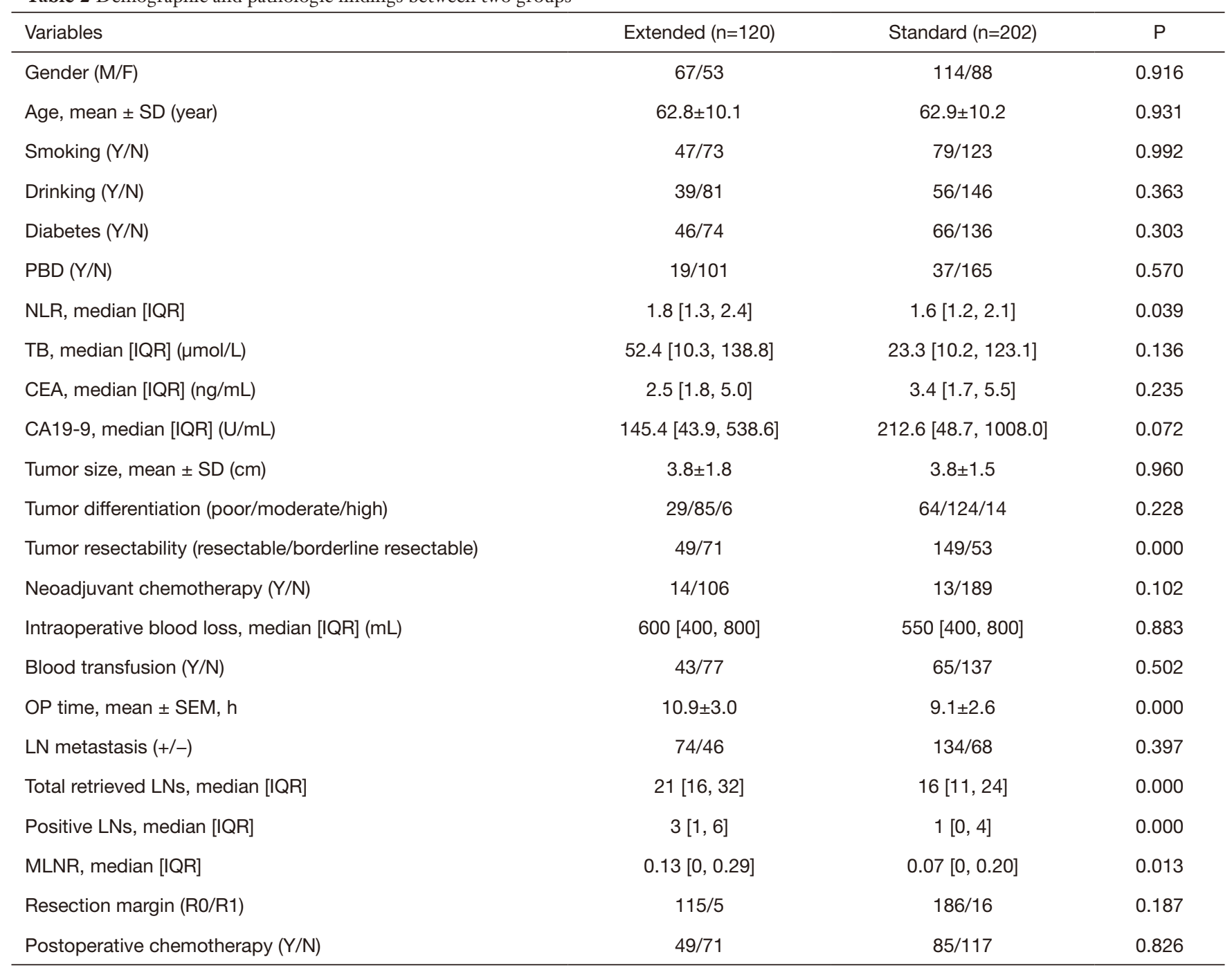

CA19-9, carbohydrate antigen 199; CEA, carcinoma embryonic antigen; IQR, interquartile range; LN, lymph node; MLNR, metastatic lymph node ratio; NLR, neutrophil to lymphocyte ratio; OP, operation; PBD, preoperative biliary drainage; TB, total bilirubin; SD, standard deviation; SEM, standard error of the mean; Y, yes; N, no.

dissection group was also higher than that in the standard lymph node dissection group $(\mathrm{P}<0.05)$. There was no significant difference in the incidence and mortality of other perioperative complications $(\mathrm{P}>0.05)$.

The median disease-free survival time of patients with extended lymph node dissection and standard lymph node dissection was 20 and 17 months, respectively, and the 1 -, 2 - and 3-year disease-free survival rates were $79.4 \%$, $41.6 \%, 32.1 \%$ and $65.7 \%, 32.9 \%$, and $22.7 \%$, respectively $(\mathrm{P}=0.205$, Figure $5 A)$. The overall median survival time of patients with extended lymph node dissection and standard lymph node dissection was 25 and 21 months, respectively, and the 1-, 2- and 3-year overall survival rates were $87.7 \%$, $60.9 \%, 33.8 \%$ and $79.1 \%, 38.9 \%, 24.6 \%$, respectively $(\mathrm{P}=0.146$, Figure $5 B)$.

\section{Comparison of patients with BRPHC by different lymph node dissection groups}

The comparison of perioperative general data between the extended dissection group and the standard dissection group was shown in Table 6, which shows that the number of lymph node dissection in the extended dissection group was more than that in the standard dissection group $(\mathrm{P}<0.05)$. 
Table 3 Morbidity and mortality between two groups

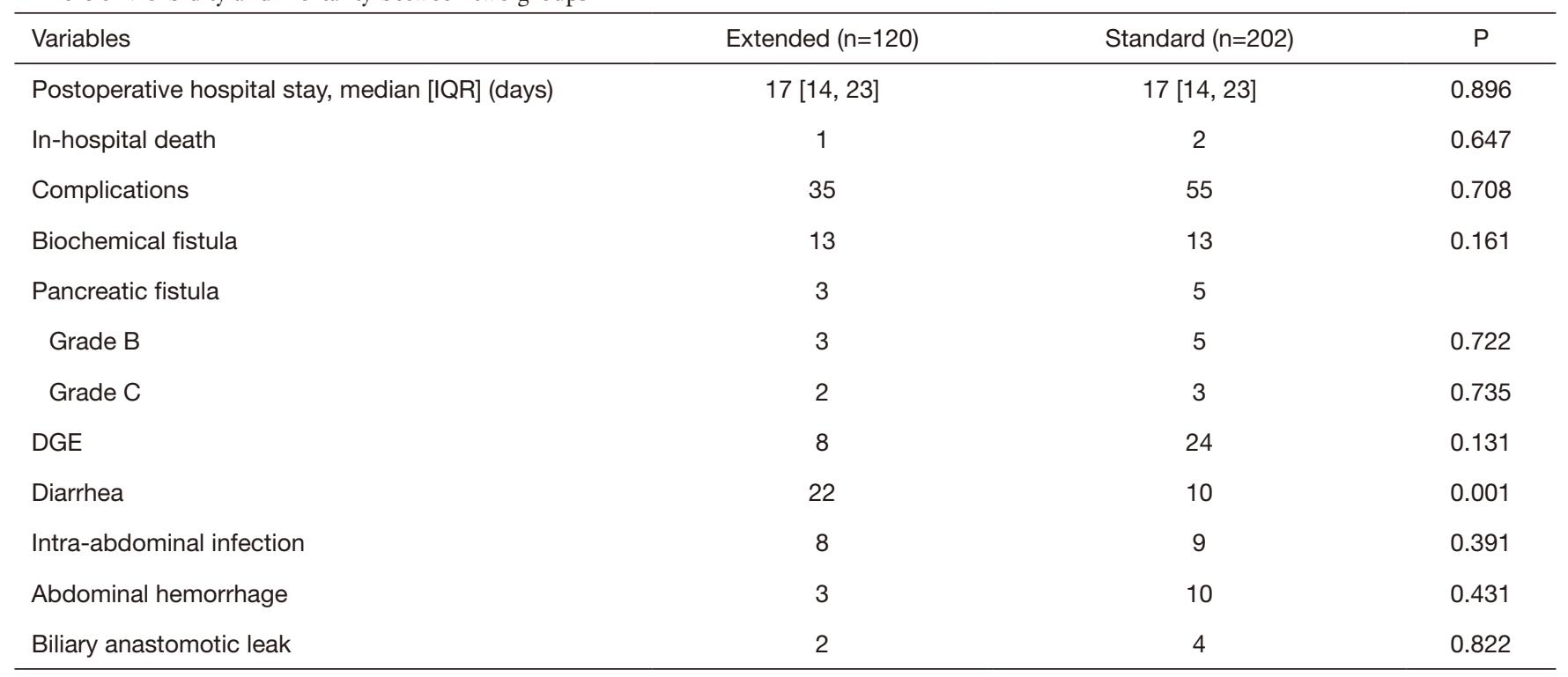

DGE, delayed gastric emptying; IQR, interquartile range.
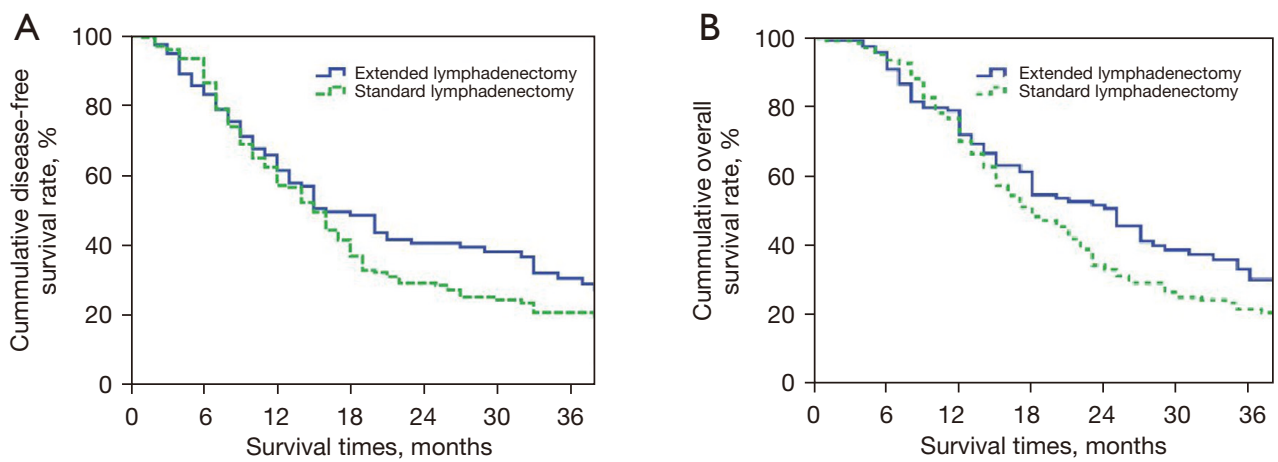

Figure 4 Overall long-term prognosis of patients with extended lymphadenectomy \& standard lymphadenectomy. (A) Disease-free survival curve between two groups; (B) overall survival curve between two groups.

The comparison of postoperative complications between the two groups was shown in Table 7. It can be seen that the incidence of diarrhea in the extended dissection group was higher than that in the standard dissection group $(\mathrm{P}<0.05)$. There was no significant difference in the incidence and mortality of other perioperative complications $(\mathrm{P}>0.05)$.

The median disease-free survival time of patients with extended lymph node dissection and standard lymph node dissection was 12 and 8 months, respectively, and the 1-, 2and 3 -year disease-free survival rates were $48.5 \%, 40.3 \%$, $26.2 \%$ and $30.4 \%, 15.6 \%, 15.6 \%$, respectively $(\mathrm{P}=0.026$, Figure $6 A$ ). The overall median survival time of patients with extended lymph node dissection and standard lymph node dissection was 18 and 12 months, respectively. The 1-, 2 - and 3-year overall survival rates were $60.7 \%, 43.3 \%, 27.4 \%$ and $43.2 \%, 17.7 \%, 17.7 \%$, respectively $(\mathrm{P}=0.007$, Figure $6 B)$.

\section{Discussion}

Lymph node status is a powerful prognostic factor in patients with pancreatic head cancer after resection as lymph node invasion occurs in a high percentage of these patients indicating poor survival (16-19). Therefore, lymphadenectomy has been recognized as an important and fundamental step during PD. A growing body of evidence shows local recurrence after conventional PD results 
Table 4 Demographic and pathologic findings between two groups in patients with RPHC

\begin{tabular}{|c|c|c|c|}
\hline Variables & Extended $(n=49)$ & Standard $(n=149)$ & $\mathrm{P}$ \\
\hline Age, mean \pm SD (year) & $65.0 \pm 9.5$ & $63.2 \pm 10.0$ & 0.255 \\
\hline Diabetes $(\mathrm{Y} / \mathrm{N})$ & $18 / 31$ & $44 / 105$ & 0.346 \\
\hline $\mathrm{PBD}(\mathrm{Y} / \mathrm{N})$ & $9 / 40$ & $30 / 119$ & 0.787 \\
\hline TB, median [IQR] $(\mu \mathrm{mol} / \mathrm{L})$ & $86.3[12.6,124.6]$ & $24.1[10.2,160.0]$ & 0.374 \\
\hline CEA, median $[\mathrm{IQR}](\mathrm{ng} / \mathrm{mL})$ & $3.1[1.3,4.4]$ & $3.4[1.6,4.8]$ & 0.229 \\
\hline CA19-9, median [IQR] (U/mL) & $145.4[60.0,324.1]$ & $188.9[47.6,648.9]$ & 0.714 \\
\hline Tumor size, mean \pm SD $(\mathrm{cm})$ & $3.6 \pm 2.1$ & $3.7 \pm 1.5$ & 0.724 \\
\hline Blood transfusion $(\mathrm{Y} / \mathrm{N})$ & $10 / 39$ & $37 / 112$ & 0.528 \\
\hline OP time, mean \pm SEM, $h$ & $9.3 \pm 2.3$ & $8.2 \pm 2.1$ & 0.004 \\
\hline LN metastasis (+/-) & $31 / 18$ & $92 / 57$ & 0.849 \\
\hline Total retrieved LNs, median [IQR] & $18[14,32]$ & $15[11,22]$ & 0.045 \\
\hline Positive LNs, median [IQR] & $1[0,3]$ & $1[0,4]$ & 0.610 \\
\hline MLNR, median [IQR] & $0.06[0,0.17]$ & $0.07[0,0.20]$ & 0.934 \\
\hline Resection margin (R0/R1) & $49 / 0$ & $140 / 9$ & 0.172 \\
\hline Postoperative chemotherapy (Y/N) & $18 / 31$ & $61 / 88$ & 0.602 \\
\hline
\end{tabular}

CA19-9, carbohydrate antigen 199; CEA, carcinoma embryonic antigen; IQR, interquartile range; LN, lymph node; MLNR, metastatic lymph node ratio; NLR, neutrophil to lymphocyte ratio; OP, operation; PBD, preoperative biliary drainage; TB, total bilirubin; SD, standard deviation; SEM, standard error of the mean; $Y$, yes; $N$, no.

Table 5 Morbidity and mortality between two groups in patients with RPHC

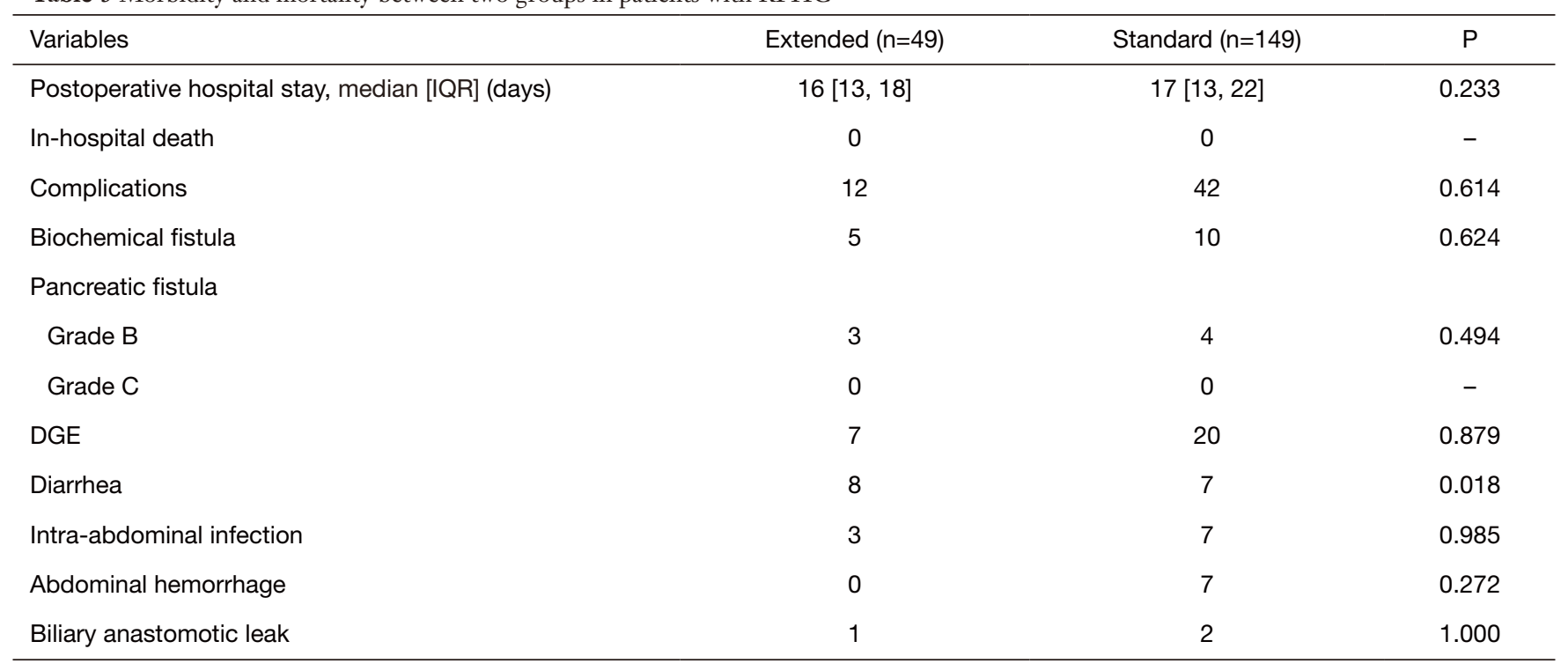

DGE, delayed gastric emptying; IQR, interquartile range; RPHC, resectable pancreatic head cancer. 

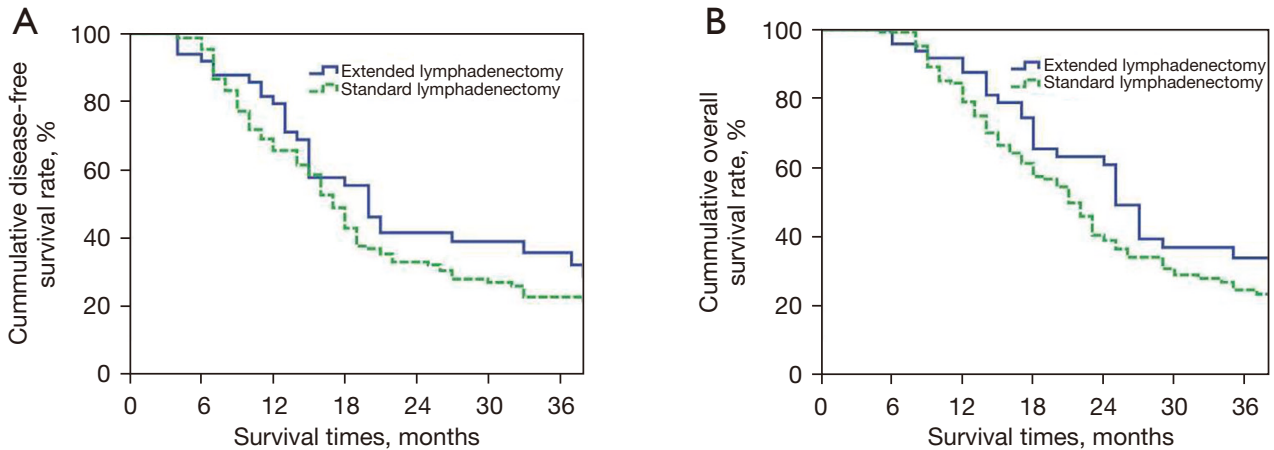

Figure 5 Overall long-term prognosis of extended lymphadenectomy \& standard lymphadenectomy in patients with resectable pancreatic head cancer. (A) Disease-free survival curve between two groups; (B) overall survival curve between two groups.

Table 6 Demographic and pathologic findings between two groups in patients with BRPHC

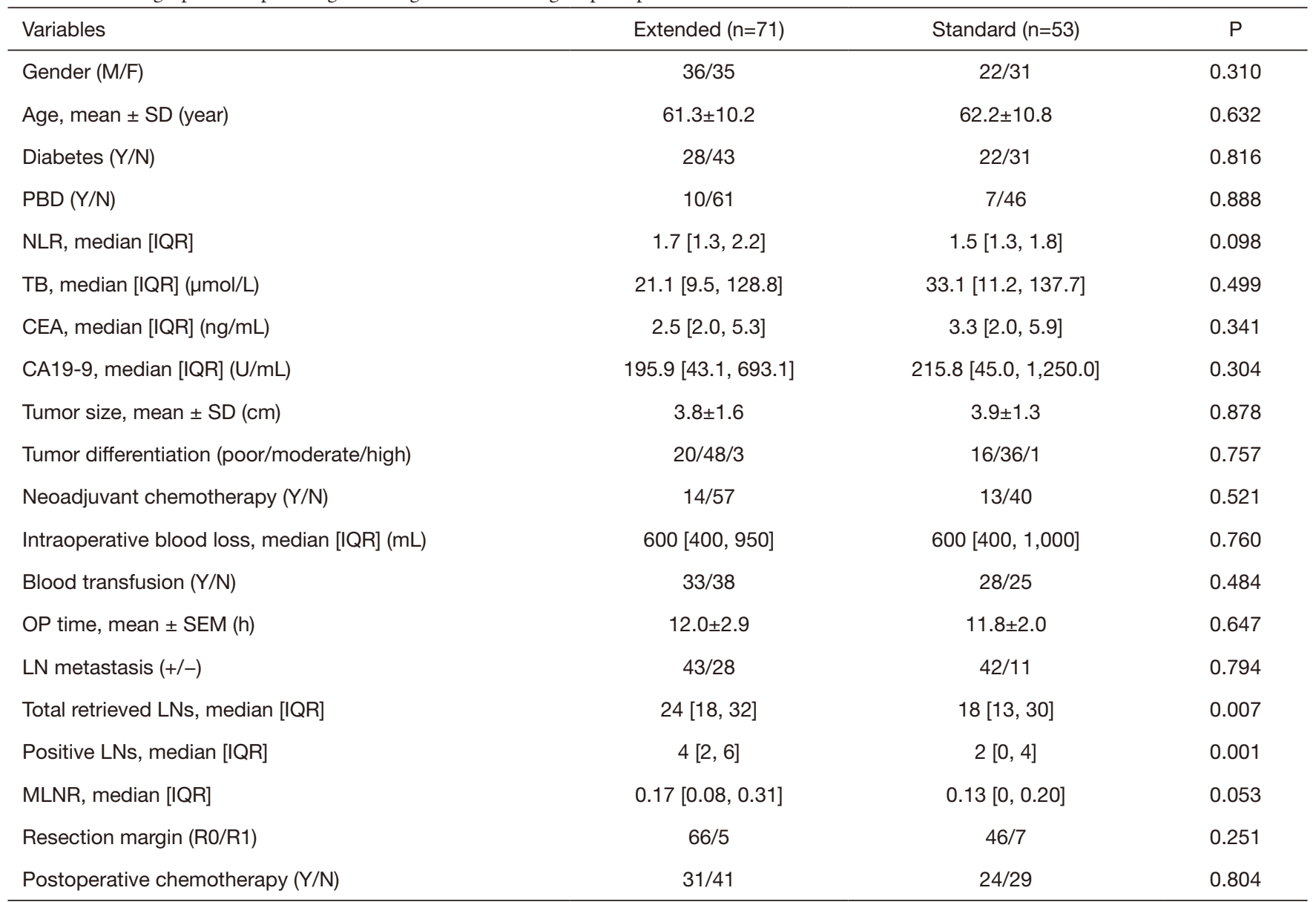

BRPHC, borderline resectable pancreatic head cancer; CA19-9, carbohydrate antigen 199; CEA, carcinoma embryonic antigen; IQR, interquartile range; LN, lymph node; MLNR, metastatic lymph node ratio; NLR, neutrophil to lymphocyte ratio; OP, operation; PBD, preoperative biliary drainage; TB, total bilirubin; SD, standard deviation; SEM, standard error of the mean; Y, yes; N, no. 
Table 7 Morbidity and mortality between two groups in patients with BRPHC

\begin{tabular}{|c|c|c|c|}
\hline Variables & Extended $(n=71)$ & Standard $(n=53)$ & $\mathrm{P}$ \\
\hline In-hospital death & 1 & 2 & 0.797 \\
\hline Complications & 23 & 13 & 0.340 \\
\hline Biochemical fistula & 8 & 3 & 0.443 \\
\hline \multicolumn{4}{|l|}{ Pancreatic fistula } \\
\hline Grade B & 0 & 1 & 0.427 \\
\hline Grade C & 2 & 3 & 0.738 \\
\hline DGE & 3 & 4 & 0.689 \\
\hline Diarrhea & 14 & 3 & 0.024 \\
\hline Biliary anastomotic leak & 1 & 2 & 0.797 \\
\hline
\end{tabular}

BRPHC, borderline resectable pancreatic head cancer; DGE, delayed gastric emptying; IQR, interquartile range.
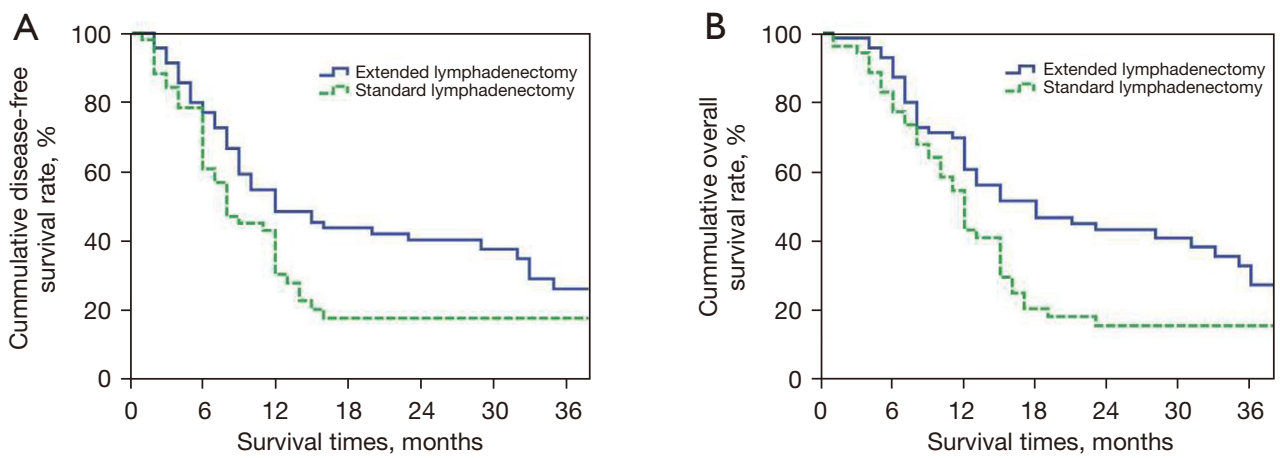

Figure 6 Overall long-term prognosis of extended lymphadenectomy \& standard lymphadenectomy in patients with borderline resectable pancreatic head cancer. (A) Disease-free survival curve between two groups; (B) overall survival curve between two groups.

from incomplete clearance of the lymph nodes suggesting extended lymphadenectomy may improve survival outcomes theoretically. For example, the survival rates after extended lymphadenectomy were reported to be greatly enhanced compared with those after standard lymphadenectomy (7,20-22). However, recent studies found that extended resection did not have survival advantages over standard resection $(8,23-25)$. Consequently, it is uncertain whether extended lymphadenectomy is superior to standard lymphadenectomy in PD.

In our study, we found extended lymphadenectomy did not improve the survival in all patients with pancreatic head cancer but to cause an increase in the difficulty of surgery (prolonged operating time) as well as in the postoperative complication (high incidence of diarrhea) due to its nature. Accordingly, the number of harvested lymph nodes was larger after extended lymphadenectomy. When we further analyzed the data merely from the subgroup of patients with resectable disease, there were no difference in survival rates between extended lymphadenectomy and standard lymphadenectomy. Therefore, comparison of the number of metastatic lymph nodes did not favor extended lymphadenectomy in patients with resectable disease.

In contrast, distinguished results with respect to the survival rates were observed in patients with BRPHC. Extended lymphadenectomy improved the disease-free 
survival and the overall survival greatly in patients with BRPHC compared with standard lymphadenectomy. BRPHC, an intermediate stage between resectable and unresectable disease, invades the mesenteric-portal or arterial axis (26). In case of superior mesenteric-portal vein involvement, venous resection and reconstruction during PD can contribute to long-term survival of these patients $(27,28)$. There has been increasing evidence indicating that patients with BRPHC could reach the survival similar to patients with resectable disease following surgery (29-31). However, few studies are reported concerning lymphadenectomy in patients with BRPHC. Actually, the reasonable extent of surgery is based on the range of lymph node invasion. The number of metastatic lymph nodes increased greatly in addition to the number of total lymph nodes in this study, which meant patients with BRPHC might have a wide range of lymph node invasion. Therefore, extended lymphadenectomy might benefit these selected patients. Since vascular resection and reconstruction can significantly affect the process, operating time was similar between the subgroups.

Currently, the risk factors for extensive lymph node metastasis remain limited. In this study, we determined large tumor size, poor tumor differentiation and BRPHC were the independent predictors favoring extended lymphadenectomy. Tumor size has been repeatedly reported to be a crucial prognostic factor for patients with pancreatic cancer (32-36), in relation to positive peritoneal lavage cytology (37) and recurrence $(38,39)$. It is thought that cells within the tumor usually acquire mutations, and finally, a sub-clone that is capable of lymph node metastasis may evolve as the primary tumor grows. Feng et al. reported nuclear-enriched abundant transcript 1 (NEAT1) could promote pancreatic cancer cell proliferation and metastasis both in vitro and in vivo associating with tumor size and lymph node (40). Oshima et al. found mutations in KRAS, p53 and SMAD4 were significantly associated with tumor size and lymph node metastasis (41). It is possible the extent of lymph node metastases tends to increase with tumor size $(42,43)$.

Another expected risk factor for extensive lymph node metastasis was the poor tumor differentiation which influenced the clinical outcome of patients. In essence, poor degree of tumor differentiation reflects rapid tumor progression (44), whereas there are rich lymphatic networks around the pancreas including the internal pancreatic lymphatics, extrapancreatic lymphatics and peripancreatic lymphatics, which make lymph node metastasis easier. Actually, differentiation of tumor cells is controlled by complex regulatory networks, which can be explained with molecular mechanism of pancreatic cancer differentiation. Xie et al. reported dysregulated KLF4 expression associated with poor differentiation of pancreatic cancer (45). Milan et al. determined FOXA2 regulated well- and poorly differentiated pancreatic ductal adenocarcinoma cells via interactions with transcription factors (46). Liang et al. found patients with poor tumor differentiation had higher MAP4K4 expression, and consequently increased number of positive lymph nodes (47). All these data support a positive association between lymph node metastasis and poor tumor differentiation.

As borderline resectability is noted as a high risk for a margin-positive status in patients with BRPHC vascular resection and reconstruction is usually required in these patients. Nevertheless, the survival still decreases with the severity of vascular involvement (25). Surprisingly, the disease-free survival and the overall survival improved in patients with BRPHC undergoing extended lymphadenectomy in our study due to a wide range of lymph node invasion. There are two possible reasons for this phenomenon. On one hand, the superior mesentericportal vein invasion might occur in patients with higher rates of poor tumor differentiation (48) as well as in patients with a larger tumor size $(49,50)$, which was also confirmed in our study; on the other hand, invasions of the plexuses around the arteries are the main causes of local recurrence after resection in these patients (51). All these suggest a higher rate of lymph node metastasis. Nevertheless, local tumor control by extended lymphadenectomy cannot overcome the negative aspects of pre-existing lymph node metastasis as well as superior mesenteric-portal vein involvement, which might explain why patients with BRPHC still have lower survival rates.

Taken together, we found that patients with BRPHC tended to have vast lymph node metastasis compared with patients with resectable disease. Extensive surgery for pancreatic carcinoma should be entertained in selected patients as it can improve their long-term survival.

The main limitation of this study is that it represented the experience of a single center. The number of patients in each subgroup is relatively small, which may limit the accuracy of our assessment. Future studies, preferably random clinical trials from multi-centers, are needed to further confirm our preliminary outcomes. 


\section{Conclusions}

Patients with BRPHC tended to have vast lymph node metastasis. Extended lymphadenectomy can improve their long-term survival, meanwhile, with regards to long term survival extended lymphnode dissection cannot compensate for missing adjuvant chemotherapy.

\section{Acknowledgments}

Funding: This study was supported by Beijing Municipal Science \& Technology Commission, PR China (grant No. Z181100001718164) and Capital's Funds for Health Improvement and Research, Beijing, China (CFH 20202-2036).

\section{Footnote}

Reporting Checklist: The authors have completed the STROBE reporting checklist. Available at https://dx.doi. org/10.21037/gs-21-201

Data Sharing Statement: Available at https://dx.doi. org/10.21037/gs-21-201

Peer Review File: Available at https://dx.doi.org/10.21037/ gs-21-201

Conflicts of Interest: All authors have completed the ICMJE uniform disclosure form (available at https://dx.doi. org/10.21037/gs-21-201). The authors have no conflicts of interest to declare.

Ethical Statement: The authors are accountable for all aspects of the work in ensuring that questions related to the accuracy or integrity of any part of the work are appropriately investigated and resolved. The study was conducted in accordance with the Declaration of Helsinki (as revised in 2013). The study was approved by the Ethics Committee of Beijing Chaoyang Hospital (No. 2020-D.309-2) and individual consent for this retrospective analysis was waived.

Open Access Statement: This is an Open Access article distributed in accordance with the Creative Commons Attribution-NonCommercial-NoDerivs 4.0 International License (CC BY-NC-ND 4.0), which permits the noncommercial replication and distribution of the article with the strict proviso that no changes or edits are made and the original work is properly cited (including links to both the formal publication through the relevant DOI and the license). See: https://creativecommons.org/licenses/by-nc-nd/4.0/.

\section{References}

1. Simard EP, Ward EM, Siegel R, et al. Cancers with increasing incidence trends in the United States: 1999 through 2008. CA Cancer J Clin 2012;62:118-28.

2. Mizrahi JD, Surana R, Valle JW, et al. Pancreatic cancer. Lancet 2020;395:2008-20.

3. O'Reilly EM, Ferrone C. Neoadjuvant or Adjuvant Therapy for Resectable or Borderline Resectable Pancreatic Cancer: Which Is Preferred? J Clin Oncol 2020;38:1757-9.

4. Nevala-Plagemann C, Hidalgo M, Garrido-Laguna I. From state-of-the-art treatments to novel therapies for advanced-stage pancreatic cancer. Nat Rev Clin Oncol 2020;17:108-23.

5. Smyth EC, Fassan M, Cunningham D, et al. Effect of Pathologic Tumor Response and Nodal Status on Survival in the Medical Research Council Adjuvant Gastric Infusional Chemotherapy Trial. J Clin Oncol 2016;34:2721-7.

6. Ghaneh P, Kleeff J, Halloran CM, et al. The Impact of Positive Resection Margins on Survival and Recurrence Following Resection and Adjuvant Chemotherapy for Pancreatic Ductal Adenocarcinoma. Ann Surg 2019;269:520-9.

7. Ishikawa O, Ohhigashi H, Sasaki Y, et al. Practical usefulness of lymphatic and connective tissue clearance for the carcinoma of the pancreas head. Ann Surg 1988;208:215-20.

8. Jang JY, Kang MJ, Heo JS, et al. A prospective randomized controlled study comparing outcomes of standard resection and extended resection, including dissection of the nerve plexus and various lymph nodes, in patients with pancreatic head cancer. Ann Surg 2014;259:656-64.

9. Pedrazzoli S, DiCarlo V, Dionigi R, et al. Standard versus extended lymphadenectomy associated with pancreatoduodenectomy in the surgical treatment of adenocarcinoma of the head of the pancreas: a multicenter, prospective, randomized study. Lymphadenectomy Study Group. Ann Surg 1998;228:508-17.

10. Tsuchiya R, Oribe T, Noda T. Size of the tumor and other factors influencing prognosis of carcinoma of the head of 
the pancreas. Am J Gastroenterol 1985;80:459-62.

11. Nagai H, Kuroda A, Morioka Y. Lymphatic and local spread of T1 and T2 pancreatic cancer. A study of autopsy material. Ann Surg 1986;204:65-71.

12. NCCN Clinical Practice Guidelines in Oncology. Pancreatic Adenocarcinoma, Version 1. 2020. Available online: https://www.nccn.org/professionals/physician_gls/ default.aspx

13. Horiguchi A, Miyakawa S, Ishihara S, et al. Gallbladder bed resection or hepatectomy of segments $4 \mathrm{a}$ and 5 for pT2 gallbladder carcinoma: analysis of Japanese registration cases by the study group for biliary surgery of the Japanese Society of Hepato-Biliary-Pancreatic Surgery. J Hepatobiliary Pancreat Sci 2013;20:518-24.

14. Clavien PA, Barkun J, de Oliveira ML, et al. The ClavienDindo classification of surgical complications: five-year experience. Ann Surg 2009;250:187-96.

15. Bassi C, Marchegiani G, Dervenis C, et al. The 2016 update of the International Study Group (ISGPS) definition and grading of postoperative pancreatic fistula: 11 Years After. Surgery 2017;161:584-91.

16. Klaiber U, Schnaidt ES, Hinz U, et al. Prognostic Factors of Survival After Neoadjuvant Treatment and Resection for Initially Unresectable Pancreatic Cancer. Ann Surg 2021;273:154-62.

17. He J, Blair AB, Groot VP, et al. Is a Pathological Complete Response Following Neoadjuvant Chemoradiation Associated With Prolonged Survival in Patients With Pancreatic Cancer? Ann Surg 2018;268:1-8.

18. Demir IE, Jäger C, Schlitter AM, et al. R0 Versus R1 Resection Matters after Pancreaticoduodenectomy, and Less after Distal or Total Pancreatectomy for Pancreatic Cancer. Ann Surg 2018;268:1058-68.

19. Kadera BE, Sunjaya DB, Isacoff WH, et al. Locally advanced pancreatic cancer: association between prolonged preoperative treatment and lymph-node negativity and overall survival. JAMA Surg 2014;149:145-53.

20. Satake K, Nishiwaki H, Yokomatsu H, et al. Surgical curability and prognosis for standard versus extended resection for T1 carcinoma of the pancreas. Surg Gynecol Obstet 1992;175:259-65.

21. Manabe T, Ohshio G, Baba N, et al. Radical pancreatectomy for ductal cell carcinoma of the head of the pancreas. Cancer 1989;64:1132-7.

22. Naganuma T, Isaji S, Kawarada Y. Staging and extended resection for pancreatic cancer. Pancreas 1998;16:355-62.

23. Farnell MB, Pearson RK, Sarr MG, et al. A prospective randomized trial comparing standard pancreatoduodenectomy with pancreatoduodenectomy with extended lymphadenectomy in resectable pancreatic head adenocarcinoma. Surgery 2005;138:618-28; discussion 628-30.

24. Michalski CW, Kleeff J, Wente MN, et al. Systematic review and meta-analysis of standard and extended lymphadenectomy in pancreaticoduodenectomy for pancreatic cancer. Br J Surg 2007;94:265-73.

25. Yeo CJ, Cameron JL, Lillemoe KD, et al. Pancreaticoduodenectomy with or without distal gastrectomy and extended retroperitoneal lymphadenectomy for periampullary adenocarcinoma, part 2: randomized controlled trial evaluating survival, morbidity, and mortality. Ann Surg 2002;236:355-66; discussion 366-8.

26. Murakami Y, Satoi S, Motoi F, et al. Portal or superior mesenteric vein resection in pancreatoduodenectomy for pancreatic head carcinoma. Br J Surg 2015;102:837-46.

27. Nakao A, Kanzaki A, Fujii T, et al. Correlation between radiographic classification and pathological grade of portal vein wall invasion in pancreatic head cancer. Ann Surg 2012;255:103-8.

28. Zhu J, Li X, Kou J, et al. Proposed Chaoyang vascular classification for superior mesenteric-portal vein invasion, resection, and reconstruction in patients with pancreatic head cancer during pancreaticoduodenectomy - A retrospective cohort study. Int J Surg 2018;53:292-7.

29. Leach SD, Lee JE, Charnsangavej C, et al. Survival following pancreaticoduodenectomy with resection of the superior mesenteric-portal vein confluence for adenocarcinoma of the pancreatic head. Br J Surg 1998;85:611-7.

30. Malleo G, Maggino L, Marchegiani G, et al. Pancreatectomy with venous resection for $\mathrm{pT} 3$ head adenocarcinoma: Perioperative outcomes, recurrence pattern and prognostic implications of histologically confirmed vascular infiltration. Pancreatology 2017;17:847-57.

31. Ravikumar R, Sabin C, Abu Hilal M, et al. Portal vein resection in borderline resectable pancreatic cancer: a United Kingdom multicenter study. J Am Coll Surg 2014;218:401-11.

32. Satoi S, Yamamoto T, Uchida K, et al. Optimal Treatment for Octogenarians With Resectable and Borderline Resectable Pancreatic Ductal Adenocarcinoma: A Multicenter Retrospective Study. Pancreas 2020;49:837-44.

33. Ueda $M$, Endo I, Nakashima $M$, et al. Prognostic 
factors after resection of pancreatic cancer. World J Surg 2009;33:104-10.

34. de Jong MC, Li F, Cameron JL, et al. Re-evaluating the impact of tumor size on survival following pancreaticoduodenectomy for pancreatic adenocarcinoma. J Surg Oncol 2011;103:656-62.

35. Petermann D, Demartines $N$, Schäfer $M$. Is tumour size an underestimated feature in the current TNM system for malignancies of the pancreatic head? HPB (Oxford) 2013;15:872-81.

36. Park H, An S, Eo SH, et al. Survival effect of tumor size and extrapancreatic extension in surgically resected pancreatic cancer: proposal for improved $\mathrm{T}$ classification. Hum Pathol 2014;45:2341-6.

37. Hirabayashi K, Imoto A, Yamada M, et al. Positive Intraoperative Peritoneal Lavage Cytology is a Negative Prognostic Factor in Pancreatic Ductal Adenocarcinoma: A Retrospective Single-Center Study. Front Oncol 2015;5:182.

38. Takahashi D, Kojima M, Sugimoto M, et al. Pathologic Evaluation of Surgical Margins in Pancreatic Cancer Specimens Using Color Coding With Tissue Marking Dyes. Pancreas 2018;47:830-6.

39. Lee JW, Kang CM, Choi HJ, et al. Prognostic Value of Metabolic Tumor Volume and Total Lesion Glycolysis on Preoperative ${ }^{18} \mathrm{~F}$-FDG PET/CT in Patients with Pancreatic Cancer. J Nucl Med 2014;55:898-904.

40. Feng Y, Gao L, Cui G, et al. LncRNA NEAT1 facilitates pancreatic cancer growth and metastasis through stabilizing ELF3 mRNA. Am J Cancer Res 2020;10:237-48.

41. Oshima M, Okano K, Muraki S, et al. Immunohistochemically detected expression of 3 major genes $(\mathrm{CDKN} 2 \mathrm{~A} / \mathrm{p} 16$, TP53, and SMAD4/DPC4) strongly predicts survival in patients with resectable pancreatic cancer. Ann Surg 2013;258:336-46.

42. Muralidhar V, Nipp RD, Mamon HJ, et al. Association Between Very Small Tumor Size and Decreased Overall

Cite this article as: Wang J, Lyu SC, Zhu JQ, Li XL, Lang R, He Q. Extended lymphadenectomy benefits patients with borderline resectable pancreatic head cancer-a single-center retrospective study. Gland Surg 2021;10(10):2910-2924. doi: 10.21037/gs-21-201
Survival in Node-Positive Pancreatic Cancer. Ann Surg Oncol 2018;25:4027-34.

43. Fortner JG, Klimstra DS, Senie RT, et al. Tumor size is the primary prognosticator for pancreatic cancer after regional pancreatectomy. Ann Surg 1996;223:147-53.

44. Panzuto F, Boninsegna L, Fazio N, et al. Metastatic and locally advanced pancreatic endocrine carcinomas: analysis of factors associated with disease progression. J Clin Oncol 2011;29:2372-7.

45. Xie VK, Li Z, Yan Y, et al. DNA-Methyltransferase 1 Induces Dedifferentiation of Pancreatic Cancer Cells through Silencing of Krüppel-Like Factor 4 Expression. Clin Cancer Res 2017;23:5585-97.

46. Milan M, Balestrieri C, Alfarano G, et al. FOXA2 controls the cis-regulatory networks of pancreatic cancer cells in a differentiation grade-specific manner. EMBO J 2019;38:e102161.

47. Liang JJ, Wang H, Rashid A, et al. Expression of MAP4K4 is associated with worse prognosis in patients with stage II pancreatic ductal adenocarcinoma. Clin Cancer Res 2008;14:7043-9.

48. Song A, Liu F, Wu L, et al. Histopathologic tumor invasion of superior mesenteric vein/ portal vein is a poor prognostic indicator in patients with pancreatic ductal adenocarcinoma: results from a systematic review and meta-analysis. Oncotarget 2017;8:32600-7.

49. Ohgi K, Yamamoto Y, Sugiura T, et al. Is Pancreatic Head Cancer with Portal Venous Involvement Really Borderline Resectable? Appraisal of an Upfront Surgery Series. Ann Surg Oncol 2017;24:2752-61.

50. Fancellu A, Petrucciani N, Porcu A, et al. The Impact on Survival and Morbidity of Portal-Mesenteric Resection During Pancreaticoduodenectomy for Pancreatic Head Adenocarcinoma: A Systematic Review and Meta-Analysis of Comparative Studies. Cancers (Basel) 2020;12:1976.

51. Sugiura T, Uesaka K, Mihara K, et al. Margin status, recurrence pattern, and prognosis after resection of pancreatic cancer. Surgery 2013;154:1078-86. 